\title{
Algoritmo de encaminamiento con reconfiguración de topología para red de sensores inalámbricos aplicada a una Microrred en modo "Isla"
}

\section{Routing Algorithm with topology reconfiguration for Wireless Sensor Network applied to microgrid in Island mode}

\author{
E.E Gaona ${ }^{1}$, P.A Mancera ${ }^{2}$, C.L Trujillo ${ }^{3}$ \\ ${ }^{1}$ GITUD, Facultad de ingenierías.Universidad Distrital Francisco José de Caldas. Email: egaona@udistrital.edu.co \\ ${ }^{2}$ Universidad Santo Tomás, Colombia.Email: pedromancera@usantotomas.edu.co \\ ${ }^{3}$ Universidad Distrital Francisco José de Caldas. Email: cltrujillo@ udistrital.edu.co
}

Recibido: may 24, 2016. Aceptado: jul 12, 2016. Versión final: jul 23, 2016

\begin{abstract}
RESUMEN
Las microrredes eléctricas son consideradas una alternativa para la generación y distribución de electricidad en entornos rurales alejados de la red de distribución principal, y necesitan nuevas estrategias de intercambio de datos entre las cargas y generadores distribuidos, según la estratégia de control elegido para la microrred, con el objetivo de entregar energía eléctrica de buena calidad. En el presente artículo se realiza el análisis de los parámetros de un algoritmo de encaminamiento por demanda, aplicado a una red de sensores inalámbricos, para el intercambio de datos de control entre los elementos que componen una microrred eléctrica aislada, con el fin de identificar y reconfigurar la topología de red para el esquema de control elegido de la microrred aprovechando los procesos de descubrimiento y búsqueda de rutas del protocolo.
\end{abstract}

Palabras Clave: AODV, Microrred, Redes de sensores inalámbricos.

\begin{abstract}
Microgrids are considered an alternative for generation and distribution of electricity in rural areas far from the main electrical grid, and new strategies for data exchange among distributed generators are needed, depending on the control scheme chosen for the microgrid, with the purpose of delivering good quality power. In this article, the parameters of an on demand routing algorithm are analyzed, applied to a wireless sensor network, for control data exchange among all islanded microgrid generators, in order to identify and reconfigure the chosen network topology scheme, for the microgrid, taking advantage of the protocol's discovery and route search processes.
\end{abstract}

Keywords: AODV, Microgrid, Wireless Sensor Network.

\section{INTRODUCCIÓN}

Las redes de sensores inalámbricos, WSN (Wireless Sensor Networks), poseen características que las posicionan entre las tecnologías más eficientes y económicas para la adquisición, procesamiento, transmisión y encaminamiento de datos, en varios campos como la domótica, la agricultura, el medio ambiente, la medicina e incluso aplicaciones militares [1]. Actualmente, conceptos como hogares, granjas o ciudades inteligentes junto al Internet de las Cosas IoT (Internet Of Things), se fundamentan en las redes de sensores inalámbricos [2].

Gracias a las tecnologías modernas de construcción de equipos electrónicos y a los avances en técnicas de transmisión y encaminamiento de datos, ambas dirigidas a un bajo consumo de energía, las redes de sensores inalámbricos son muy usadas por su transmisión

Este artículo puede compartirse bajo la licencia CC BY-ND 4.0 (https://creativecommons.org/licenses/by-nd/4.0/) E.E Gaona, P.A Mancera, C.L Trujillo. "Algoritmo de encaminamiento con reconfiguración de topología para red de sensores inalámbricos aplicada a una Microrred en modo "Isla", UIS Ingenierías, vol. 15, no. 2, pp. 93-104, jul-dic 2016,doi:10.18273/revuin.v15n2-2016008. 
confiable de datos, con bajos costos económicos y un consumo de energía casi imperceptible [3],[4].

Una de las aplicaciones que tienen las redes de sensores inalámbricos es el intercambio de datos de control, especialmente en sistemas compuestos por múltiples dispositivos, en su mayoría dispersos sin una ubicación geográfica definida, que necesitan comunicarse entre ellos, para mantener una gestión continua de todas las variables y recursos, y una adecuada toma de decisiones.

Un ejemplo de este tipo de aplicación está en las microrredes eléctricas, donde las fuentes de energía, en su mayoría renovables, cargas distribuidas y baterías, deben coordinarse para mantener unos niveles de tensión, frecuencia y potencia estables, adecuados para suministrar electricidad a un entorno rural aislado de la red de distribución principal, por lo que deben funcionar de forma autónoma o en modo "Isla", haciendo que su control y gestión posean características particulares que deben tenerse en cuenta en los esquemas de control [5].

El sistema eléctrico en Colombia, muestra la existencia de un gran número de Zonas No Interconectadas (ZNI) que requieren apoyo por parte del gobierno nacional, para poder extender el servicio eléctrico a las zonas apartadas e incluir los sistemas compuestos de fuentes de energía renovable. En [6] se muestra el panorama de las microrredes rurales en Colombia, identificando sus problemáticas como la falta de infraestructura para cubrir el territorio nacional, los fenómenos climáticos impredecibles, los problemas de orden público y la ausencia de una definición técnica y legal para su despliegue.

Los esquemas de control para microrredes modo "Isla" requieren de un intercambio continuo de valores de variables como tensiones, frecuencias, corrientes y potencias, entre sus componentes y de esta forma estabilizar fluctuaciones para lograr mantener la calidad de la energía, especialmente en horas pico donde el consumo es variado e impredecible [7]. Este intercambio de datos se puede realizar a través del despliegue de una red de sensores, cuyos nodos estarían distribuidos en los generadores, las cargas y los buses, para el sensado de todas las variables y su encaminamiento hacia el nodo central.

Por lo anterior, se propone un modelo del sistema de comunicaciones de una microrred en modo "Isla" utilizando un algoritmo de encaminamiento que permita reconfigurar su topología de red, conforme al esquema de control seleccionado para la microrred, generando, un ahorro en tiempos de procesamiento y mayor eficiencia en el intercambio de datos de control y la toma de decisiones.

En la sección 2, se muestran las características generales de las redes de sensores inalámbricos, haciendo un énfasis en los algoritmos de encaminamiento por demanda. Las generalidades de las microrredes se mencionan en la sección 3, junto a los esquemas de control más usados, especificando las necesidades de intercambio de datos de control para mantener una eficiencia energética apropiada. La sección 4 muestra la metodología del trabajo propuesto del sistema de comunicaciones de una microrred aislada, enfocándose en el algoritmo de encaminamiento que se desea usar para mejorar la eficiencia en el intercambio de datos, junto a un análisis de resultados en la sección 5 Finalmente, la sección 6 muestra las conclusiones y trabajos futuros.

\section{REDES DE SENSORES INALÁMBRICOS}

\subsection{Características generales de una red de sensores inalámbricos (WSN)}

Una red de sensores inalámbricos, es un conjunto de dispositivos con capacidad de sensado, procesamiento y transmisión de datos, llamados "Nodos Sensores", que se despliegan en grandes o pequeñas cantidades, en posiciones fijas o de forma aleatoria en distintos entornos geográficos, para la recolección y transmisión de datos en un área determinada o para la toma de decisiones en un sistema compuesto de dispositivos que deban controlarse según su comportamiento [8], [9]. Las capas que intervienen en la implementación de este tipo de redes se describen a continuación [10]:

2.1.1 Capa de aplicación. Es en esta capa se encuentran los distintos programas diseñados para el manejo de la red, al nivel del usuario. La capa de aplicación depende del fabricante de la tecnología de los sensores inalámbricos, y se encarga de los procesos de sensado y cifrado, junto al procesamiento y almacenamiento de los datos obtenidos.

2.1.2 Capa de transporte. Mantiene el flujo de datos de acuerdo a las necesidades de la red. Se aplican los protocolos orientados y no orientados a la conexión.

2.1.3 Capa de red. Se encarga de encaminar los datos provenientes de la capa de transporte, estableciendo las rutas adecuadas para el intercambio de información. Su importancia radica en el algoritmo de encaminamiento, 
de acuerdo a las necesidades y prioridades de la red, como el mantener una óptima calidad de servicio, el ahorro de energía en los sensores y el tiempo de vida.

2.1.4 Capa de enlace de datos. Capa que permite a los nodos, acceder a los canales o medios de transmisión, evitando colisiones, gracias a los servicios de control de acceso al medio, y detección y corrección de errores, en el envío de tramas.

2.1.5 Capa de física. El estándar IEEE 802.15.4, cumple con los requerimientos de transmisión para una red de sensores inalámbricos: Una baja tasa de transmisión de datos y costos de implementación reducidos, usando una frecuencia de $2,4 \mathrm{C}$ potencias de $1 \mathrm{~mW}$ [11]. Sin embargo, la capa fí puede implementar sobre otras tecnologías, como Wi.Fi, dependiendo de las necesidades de la aplicación, y las limitaciones y ventajas de cada tecnología.

Los nodos sensores, están ligados a protocolos y algoritmos de configuración y encaminamiento para poder coordinar la red y transmitir la información entre ellos o hacia un nodo central. Según la aplicación de la red, se pueden proponer distintos tipos de algoritmos de encaminamiento, siempre enfocados a mejorar el tiempo de vida en aspectos como la velocidad y los tiempos de transmisión, junto a un consumo eficiente de energía de los nodos [12]-[13].

\subsection{Algoritmos de encaminamiento en redes de sensores inalámbricos}

Dependiendo de la funcionalidad de los dispositivos que intervienen en la topología de una red de sensores, puede definirse si se trabaja en conexión tipo malla, estrella o árbol (Cluster - Tree) como se muestra en la figura 1.

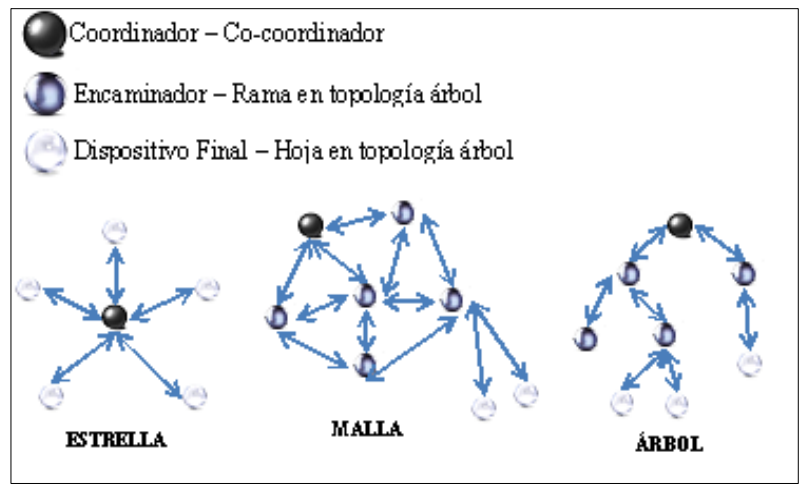

Figura 1. Topologías en redes de sensores inalámbricos [3] [14].

Fuente. Elaboración propia
Al definirse la topología, se debe tener en cuenta el tipo de algoritmo de encaminamiento a implementarse, para que cumpla con las funcionalidades de descubrimiento de rutas, establecimiento de enlaces, control del envío y deteccion de errores. Entre los algorimos más usados para redes de tipo Ad-hoc, se encuentran DYMO (Dynamic Manet On-demand) y AODV (Ad Hoc Ondemand Distance Vector), este último elegido para la implementación del modelo de comunicaciones de la microrred [15].

Teniendo en cuanta que DYMO es sucesor de AODV, ambos algoritmos poseen fortalezas y debilidades, en parámetros relacionados con la eficiencia de una red de sensores inalámbricos, como la colisión de paquetes, el overhead y el throughput, sobre entornos de simulación exigentes respecto a la movilidad y número de nodos. [16],[17]

AODV es un algoritmo de encaminamiento por demanda que establece un enlace entre un nodo origen y un nodo destino, unicamente cuando necesita enviar información, y desecha la ruta al terminar el intercambio, generando una nueva conexión en caso de otro envío. AODV trabaja con tres tipos de mensajes enviados via UDP y con encabezados IP, utilizados para el proceso de establecimiento de un enlace [17]:

- Route Request (RREQ) : Generado por el nodo origen $\mathrm{y}$ reenviado a través de los nodos intermedios hacia el nodo destino. Permite un descubrimiento de posibles caminos hacia el destino.

- Route Reply (RREP): Mensaje generado desde el destino hacia el origen a través de una sola ruta, elegida para la transmisión.

- Route Error (RERR): Mensaje de error que se genera cuando un enlace se interrumpe.

En una red Ad-Hoc basada en el algoritmo de encaminamiento por demanda AODV, los mensajes de solicitud, respuesta y error (RREQ, RREP, RERR) se controlan por medio de variables y banderas, incluidas en dichos mensajes, asegurando que no haya redundancia, ciclos sin fin $y$ gasto innecesario de recursos [18].

Entre los campos que componen los mensajes de solicitud y respuesta (RREQ y RREP), se resaltan los siguientes:

- Sequence Destination Number: Campo que contiene un número de secuencia de los paquetes reenviados hacia el destino, con el fin de evitar paquetes duplicados. 
- Precursor list (lista de vecinos): Contiene la dirección IP de los vecinos directos de un nodo.

- Hop counter: Contador de saltos muy usado en algoritmos de encaminamiento.

- $\quad$ TTL: Tiempo de vida del paquete.

- Direcciones de origen y destino: Direcciones IP, versión 4.

El algoritmo DYMO (Dynamic MANET On-demand), se basa en los mismos tipos de mensajes de solicitud, respuesta y error que en AODV. La principal diferencia radica en que cada nodo intermedio, almacena una ruta hacia el nodo origen, reduciendo el numero de paquetes de control (overhead), por lo que se requieren nodos de mayor capacidad de almacenamiento y procesamiento.Dymo reduce el overhead, pero aumenta el tamaño de los paquetes.

Por lo anterior, se concluye que las carateristicas del algoritmo AODV, permiten un intercambio de datos eficiente, en aplicaciones de intercambio de datos de control, con nodos menos costosos y con tamaños reducidos de paquetes, que contienen la información relevante que proviene de los generadores distribuidos en las microrredes eléctricas [19].

\section{APLICACIÓN DE REDES DE SENSORES INALÁMBRICOS EN MICRORREDES ELÉCTRICAS}

Las microrredes, son consideradas una alternativa a la generación y distribución de energía eléctrica en entornos rurales donde la red de distribución principal no alcanza a llegar [6]. Están compuestas por un conjunto de generadores de combustibles fósiles o de energías renovables de tipo eólico o solar, que producen electricidad generalmente a niveles de media o baja tensión. Debido a que la energía proveniente de los generadores es intermitente, especialmente en las renovables, además de que existen microrredes que manejan tensiones AC, DC o híbridas, se deben incluir convertidores, inversores y elementos de almacenamiento (banco de baterías), que permitan alimentar de manera adecuada a las cargas conectadas. Estos generadores, interconectados de forma adecuada y con un sistema de control confiable, pueden generar energía en un área específica, e incluso dar soporte a la red principal, si tiene la posibilidad de conectarse [20][21]. En la figura 2 se muestra el esquema general de una microrred.

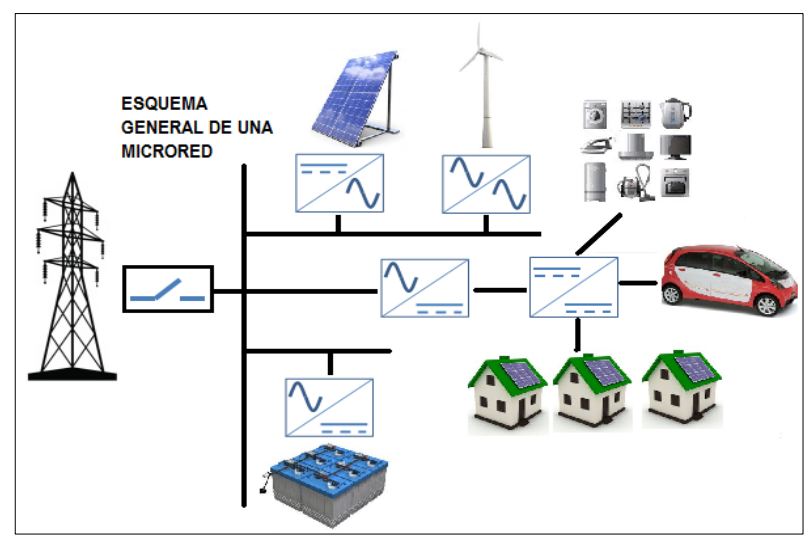

Figura 2. Modelo general de una microrred Fuente. Elaboración propia

Los esquemas de control para microrredes, definen los parámetros que deben intercambiarse entre sus elementos y las técnicas de compensación en las salidas de cada generador, para mantener los niveles deseados de tensiones, corrientes y potencias, en casos de fallas o manejar los máximos consumos de las cargas [22].

Una microrred en modo "isla", funciona totalmente independiente de la red principal, lo cual lleva a asuntos económicos y técnicos que se deben manejar, como la calidad de la energía, la regulación de la tensión, la estabilidad de la red, los armónicos, la confiabilidad, protección y control. Los dos factores que deben controlarse en una microrred aislada, para mantener sus valores dentro de los límites aceptados son la frecuencia y la tensión. Aparecen estrategias de control que gestionan variables como las potencias real y reactiva, las distintas cargas según su prioridad, los niveles y tipos de tensión en buses de alimentación y los sistemas de almacenamiento [23]. Para implementar un esquema de control, se debe tener en cuenta los siguientes factores:

- Control de la tensión y la frecuencia.

- Equilibrio entre generación y demanda.

- Calidad de la Potencia de salida.

- Las fuentes de energía.

- La comunicación entre los componentes de la microrred.

- La microrred debe planearse para modo "isla".

- Gestión de las cargas según la demanda "Load Shedding".

Según Vandoorn [24], las estrategias de control pueden depender o ser independientes de un sistema de comunicación entre sus elementos. En estos últimos, cada generador es autónomo, y su estabilidad depende 
TABla I

ESTRATEGIAS DE CONTROL CON COMUNICACIÓN

\begin{tabular}{|c|c|}
\hline \multicolumn{2}{|c|}{ DESCRIPCIÓN DE ESQUEMAS DE CONTROL EN MICRORREDES } \\
\hline $\begin{array}{l}\text { ESQUEMAS DE CONTROL } \\
\text { CON COMUNICACIÓN }\end{array}$ & DESCRIPCIÓN \\
\hline Control Central & $\begin{array}{l}\text { Un coordinador central gestiona todas las interfaces electrónicas de la } \\
\text { microrred para mantener el balance de potencias } \mathrm{P} \text { y } \mathrm{Q} \text {. Se necesita un enlace } \\
\text { de comunicaciones entre el coordinador y cada unidad. Se usan algoritmos } \\
\text { sencillos de control en los convertidores. }\end{array}$ \\
\hline Maestro/Esclavo (M/E) & $\begin{array}{l}\text { Una unidad es asignada como módulo maestro, para la regulación de tensión } \\
\text { de salida y especificar la corriente de referencia para cada inversor. El resto de } \\
\text { unidades son esclavas, encargadas de lograr una distribución de corriente } \\
\text { equitativa, según las órdenes y datos enviados por el maestro. }\end{array}$ \\
\hline $\mathrm{M} / \mathrm{E}$ sin controlador central. & $\begin{array}{l}\text { Un módulo maestro y el resto esclavos. No existe controlador central. El } \\
\text { maestro controla la tensión de las cargas y mide la corriente total de la misma } \\
\text { para elegir el valor de la corriente que debe tener cada esclavo, actúan como } \\
\text { fuentes de corriente y garantizan el valor establecido por el maestro. }\end{array}$ \\
\hline M/E con controlador central. & $\begin{array}{l}\text { El valor de la tensión de referencia no se transmite, por lo que el maestro ya } \\
\text { no provee el valor de la corriente para los esclavos. Esta tarea la realiza una } \\
\text { unidad de control central que calcula la corriente y envía este dato a los } \\
\text { esclavos. }\end{array}$ \\
\hline $\begin{array}{l}\text { división de corriente } \\
\text { (Instantánea o promedio). }\end{array}$ & $\begin{array}{l}\text { Estrategia de control que depende de la comunicación entre módulos, donde } \\
\text { por medio de un bus dedicado a la distribución de corriente y señales de } \\
\text { sincronización, permite determinar la desviación de la corriente de salida y } \\
\text { llevarla al valor deseado. }\end{array}$ \\
\hline Control de cadena circular. & $\begin{array}{l}\text { En este esquema, los módulos están interconectados en una configuración } \\
\text { circular, donde cada módulo está pendiente de la corriente del módulo } \\
\text { anterior, logrando su distribución igualitaria. Cada módulo controla su propia } \\
\text { tensión de salida para mantenerlo en el valor de referencia. }\end{array}$ \\
\hline Control distribuido. & $\begin{array}{l}\text { Uso de sistemas de comunicaciones de poco ancho de banda, para la conexión } \\
\text { entre módulos con el fin de mantener una distribución adecuada de potencia } \\
\text { instantánea y mantener la calidad al momento de alimentar las cargas. }\end{array}$ \\
\hline
\end{tabular}

Fuente: [24], [26] 
TABlaII

Comparacion Software de simulación de redes

\begin{tabular}{|l|l|}
\hline \multicolumn{2}{|c|}{ SIMULADORES DE REDES } \\
\hline SIMULADOR & \multicolumn{1}{|c|}{ GENERALIDADES } \\
\hline OPNET & $\begin{array}{l}\text { Comercial. Simulador de eventos discreto modelado Orientado a Objetos, sin } \\
\text { acceso al código, Interfaz gráfica poderosa, soporte para simulaciones } \\
\text { inalámbricas. }\end{array}$ \\
\hline $\begin{array}{l}\text { NETWORK SIMULATOR 2 } \\
\text { NS2 }\end{array}$ & $\begin{array}{l}\text { Libre. Simulador de eventos discreto modelado Orientado a Objetos. } \\
\text { Planificador de Eventos; usa dos lenguajes: C++ (que provee desempeño en } \\
\text { la ejecución) y OTCL (Object tool Command language) apropiado para } \\
\text { escribir código de simulación; Posee algunas extensiones de simulación para } \\
\text { WSN. SensorSim y NRL. }\end{array}$ \\
\hline $\begin{array}{l}\text { NETWORK SIMULATOR 3 } 3 \text { NS3 } \\
\text { JAVA SIMULATOR }\end{array}$ & $\begin{array}{l}\text { Libre. Dirigido a propósitos educativos; Usa lenguajes de programación C++ } \\
\text { y Phyton, permite integración con otro software de redes tipo open source; } \\
\text { Soporte para virtualización, acercamiento a redes reales, No es compatible } \\
\text { con NS2. }\end{array}$ \\
\hline OMNET++ & $\begin{array}{l}\text { Libre. Orientado a Componentes; Basado en ACA (Autonomous Component } \\
\text { Architecture) concepto de componente y puerto Java y OTLC; Interface que } \\
\text { permite integración con otros lenguajes de secuencia de comandos (script) } \\
\text { como Perl, TCL o Python; Posee un framework o modelo de simulación } \\
\text { WSN. }\end{array}$ \\
\hline
\end{tabular}

Fuente. [27]-[29].

de técnicas como el control "droop" [25], donde se busca controlar la tensión y la frecuencia, a partir de un ajuste de las potencias activa y reactiva de salida. Las estrategias basadas en sistemas de comunicación, necesitan mediciones e intercambio de datos entre sus elementos para que las variables que sirven de referencia como las corrientes, tensiones y potencias lleguen a su destino en el menor tiempo posible. La tabla I muestra una descripción general de las diferentes estrategias dependientes de la comunicación, evidenciando la necesidad del uso de las redes de sensores inalámbricos para transferencia de datos de control [24], [26].

\section{METODOLOGÍA}

El algoritmo de encaminamiento por demanda AODV con capacidad de reconfigurar la topología, hace parte de un sistema de comunicaciones para la transferencia de datos de control de cada generador distribuido, en una microrred aislada, para adaptarse al esquema de control, a través de una red de sensores inalámbricos.

\subsection{Software de Simulación}

Se realizó una revisión de las características principales de las herramientas de simulación de redes, con el fin de seleccionar la indicada para cumplir con los requerimientos del sistema de comunicaciones e intercambio de datos de control. La tabla II, menciona los simuladores más usados y sus generalidades [27][29]. 
La tabla III, muestra una comparación de las carácterísticas de los simuladores relacionados en la tabla II, que permiten justificar la escogencia de la herramienta de simulación a usar con la red de sensores propuesta.

OMNET++, es un entorno de simulación de redes, basado en Eclipse $(\mathrm{C}++)$, de eventos discretos, con un conjunto de librerías especializadas para todas las capas del modelo OSI, incluidas en marcos de trabajo como INET y MIXIM, permitiendo trabajar directamente sobre el algoritmo de encaminamiento y los paquetes que se envían, en los distintos tipos de redes Ad-Hoc, especialmente de sensores inalámbricos [30].

Comparado con los simuladores listados en la tabla II, Omnet++, posee un entorno de desarrollo integrado basado en Eclipse, con interfaz gráfica robusta y librerías para animaciones, que facilítan el seguimiento de los parámetros de la red, en tiempo de ejecución.

Omnet++, se caracteriza por tener un lenguaje de descripción de redes NED (NEtwork Description), que permite discriminar los modelos de configuración de la red y las clases que los controlan, lo que permite una mejor organización de los distintos niveles y jerarquías de la simulación.

Simuladores comerciales como OPNET, no permiten el acceso al código, limitando la capacidad de edición en aspectos como el protocolo de encaminamiento o los campos de los paquetes que se quieren transmitir. Sin embargo, se reconocen las ventajas en su interfáz gráfica, el soporte por su licencia y su versión académica que permite trabajar en proyectos a nivel universitario [31].

\subsection{Entorno de Simulación}

Aplicando las librerías especializadas de OMNET++, se propone un entorno de simulación que maneje las variables mencionadas anteriormente y permita analizar la eficiencia del algoritmo en dos casos particulares:

- El primer caso, consiste en modificar el número de nodos sensores intermedios entre el origen y el destino para analizar el número de paquetes que se transmiten, midiendo su eficiencia con cantidades crecientes de nodos, mostrando su escalabilidad al momento de agregar nuevos nodos para los generadores y cargas distribuidas en las microrred.

- En el segundo caso, se inhabilitan nodos sensores por medio de un archivo de configuración de extensión XML, simulando la desactivación de algunos generadores distribuidos que permiten emular fallos o desactivación del generador. Los resultados permiten evidenciar la función de las variables y banderas del algoritmo para modificarlos y reconfigurar la topología.

- TABla III

- Características Omnet++

\begin{tabular}{|c|c|c|c|c|c|}
\hline \multicolumn{6}{|c|}{$\begin{array}{l}\text { CUADRO COMPARATIVO HERRAMIENTAS DE } \\
\text { SIMULACIÓN }\end{array}$} \\
\hline PARÁMETRO & OPNET & NS2 & NS3 & JAVA S. & $\begin{array}{c}\text { OMNET+ } \\
+\end{array}$ \\
\hline $\begin{array}{l}\text { Lenguaje } \mathrm{C}++, \\
\text { basado en } \\
\text { componentes, } \\
\text { modular }\end{array}$ & SI & SI & SI & NO & SI \\
\hline Software Libre. & NO & SI & SI & SI & SI \\
\hline $\begin{array}{l}\text { Lenguaje de } \\
\text { Descripción de } \\
\text { redes "NED" }\end{array}$ & NO & NO & NO & NO & SI \\
\hline $\begin{array}{c}\text { Interfaz gráfica } \\
\text { de usuario } \\
\text { robusta para } \\
\text { animaciones. }\end{array}$ & SI & NO & NO & NO & SI \\
\hline $\begin{array}{c}\text { Librerías para } \\
\text { todas las capas } \\
\text { del modelo } \\
\text { OSI }\end{array}$ & SI & SI & SI & SI & SI \\
\hline $\begin{array}{l}\text { Librerías para } \\
\text { almacenar } \\
\text { datos } \\
\text { estadísticos } \\
\end{array}$ & NO & NO & NO & NO & SI \\
\hline $\begin{array}{c}\text { Archivos de } \\
\text { almacenamient } \\
\text { o de resultados } \\
\text { (Histogramas) } \\
\end{array}$ & NO & NO & NO & NO & SI \\
\hline $\begin{array}{l}\text { Marcos de } \\
\text { Trabajo } \\
\text { especializados } \\
\text { en redes de } \\
\text { sensores }\end{array}$ & SI & SI & SI & SI & SI \\
\hline
\end{tabular}

$\bullet$

OMNET++ posee librerías y comandos que almacenan cualquier tipo de variables de tipo contador, en archivos de extensión .SCA y .VEC para visualizarse como gráficos de línea o histogramas. Para este caso, se visualizan los resultados de los paquetes transmitidos y rechazados. 


\subsection{Topología de la red de comunicaciones}

El planteamiento de reconfiguración de topología se basa en un conteo de los vecinos de cada nodo y la funcionalidad que tienen para poder deducir automaticamente la topología en la que opera, para esto se requiere del conocimiento de los campos del mensaje de solicitud de ruta RREQ y de respuesta RREP, para luego modificarse. Lo anterior requiere un analisis del desempeño de las redes AODV tradicionales para estimar, la viabilidad de la modificación, a nivel de parametros como el BER (Bit Error Rate), y los paquetes descartados, haciendo necesario el uso de un software de simulación basado en un lenguaje de programación como java o $\mathrm{C}++$.

\subsection{Casos simulados}

El diseño de una microrred, debe contemplar la posibilidad del aumento en la cantidad de generadores y cargas distribuidas que la componen, por ejemplo para los casos en que aumenta la demanda de energía electrica.

TABLA IV

\section{CONDICIONES DE SIMULACIÓN AODV}

\section{CONFIGURACIÓN INICIAL}

Numero de nodos: 15

Tamaño de paquetes RREQ y RREP: 24 bytes

Tamaño de paquetes de información: 60 bytes

Área: 1000 x1000 metros cuadrados

Separación promedio entre nodos 50 metros

Tiempo de simulación: 10 segundos

Resultados a obtener: Tasa de error de bits a nivel de capa física y paquetes descartados.

Topología: Malla

Fuente. Elaboración propia

El número de nodos sensores puede incrementarse junto a los mensajes de control, por lo que el algoritmo de encaminamiento, debe ser eficiente en el intercambio de paquetes, razón por la cual se proponen dos escenarios de simulación, para una red de sensores, aplicando el algoritmo AODV sobre el estandar IEEE 802.11, con el fin de realizar mediciones de los paquetes descartados a nivel de capa de red y de la tasa de error de bit a nivel de capa física [32].

En el primer caso de simulación, el nodo destino (host 5), representa una fuente de energía o generador distribuído, y se encuentra dentro del alcance del nodo origen (host 0 ), el cual representa el nodo controlador de la micrrorred, como se muestra en la figura 3.
En el segundo caso, el nodo destino (host 2), se encuentra fuera del alcance del orígen (host 0), requieriendo saltos a través de nodos intermedios para recibir la información (figura 4). En la tabla IV, se muestran las condiciones y configuraciones iniciales de simulación para ambos casos.

\section{RESULTADOS}

Para modificar los campos de los mensajes de solicitud y respuesta RREQ y RREP e interpretar la topología, se realiza el análisis del protocolo AODV, a través de los resultados obtenidos en las simulaciónes, observando la eficiencia de la red al momento de generar e intercambiar mensajes de solicitud y respuesta RREQ y RREP, y poder hacer una estimación del costo en transmision.

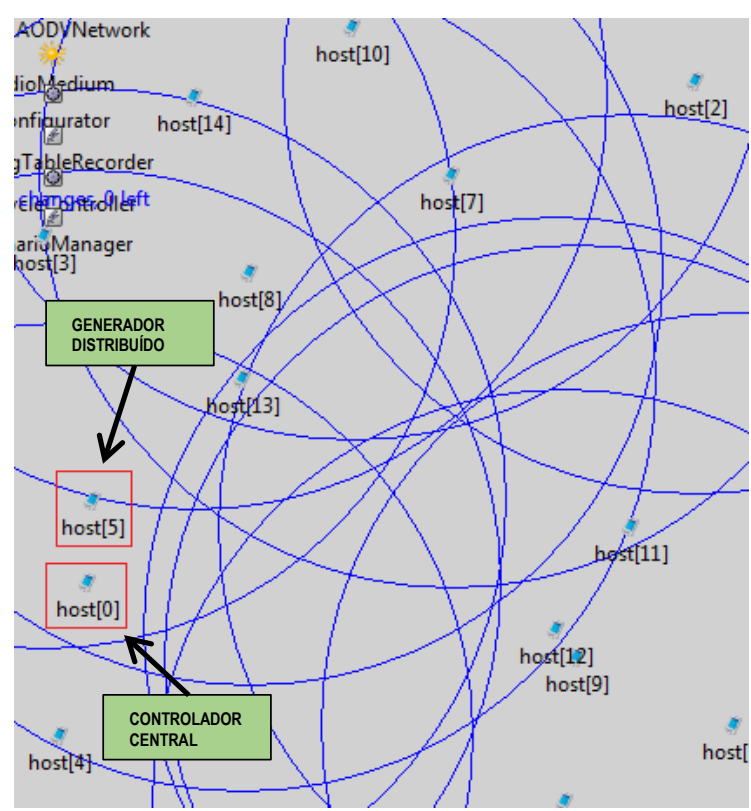

Figura 3. Caso 1 de simulación desde el nodo origen (host 0 ) con ruta directa al nodo destino (host 5)

Fuente. Elaboración propia 


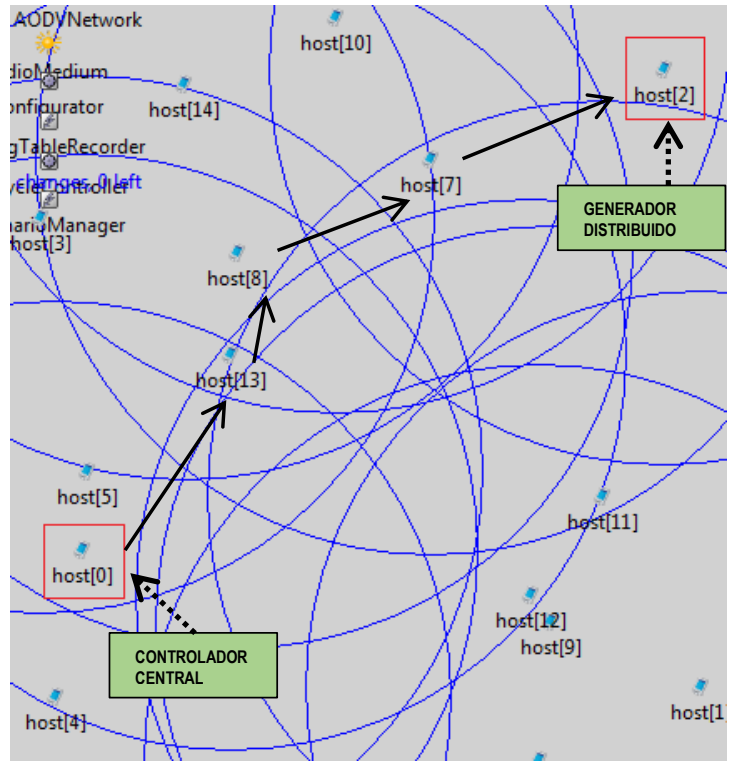

Figura 4. Caso 2 simulación origen (host 0 ) con destino a un nodo (host 2) fuera de su alcance.

Fuente. Elaboración propia

\subsection{Caso 1. Nodo destino al alcance del nodo origen}

En la tabla $\mathrm{V}$ se recopilan los resultados obtenidos en las simulaciones, respecto al número de bits erróneos (BER), junto al número de paquetes recibidos y aceptados por cada host AODV, en un intervalo de tiempo de 10 segundos, mientras el algoritmo AODV realiza su proceso de descubrimiento de ruta y el envío de datos entre el nodo orígen y destino. La figura 5 muestra las gráficas para los paquetes recibidos exitosos y el BER para cada nodo.

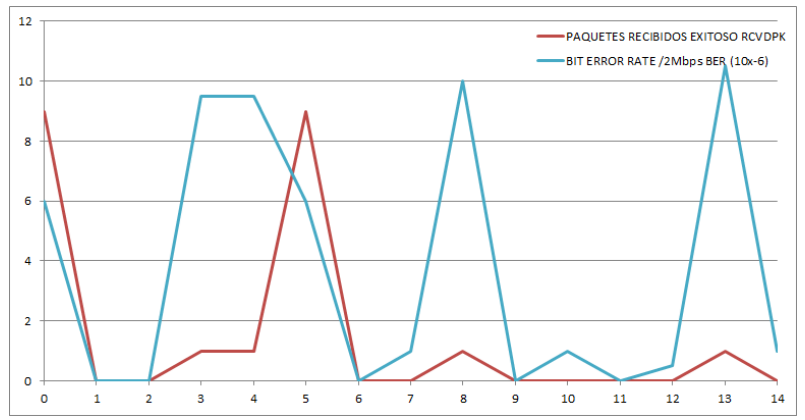

Figura 5. Caso 1, paquetes recibidos exitosos y "BER" por nodo. Fuente. Elaboración propia
TABLA V

RESULTADOS CASO 1 NODO DENTRO DEL ALCANCE DEL ORÍGEN

\begin{tabular}{|c|c|c|c|c|}
\hline \multicolumn{5}{|c|}{$\begin{array}{l}\text { NODO DENTRO DEL ALCANCE } \\
\text { (host } 0 \text { a host 5) }\end{array}$} \\
\hline \multirow{2}{*}{$\begin{array}{l}\text { HOST } \\
\text { AODV }\end{array}$} & \multirow{2}{*}{$\begin{array}{c}\text { PAQUETES } \\
\text { RECIBIDOS } \\
\text { EXITOSO } \\
\text { RCVDPK }\end{array}$} & \multirow{2}{*}{$\begin{array}{l}\text { PAQUETES } \\
\text { RECIBIDOS }\end{array}$} & \multicolumn{2}{|c|}{$\begin{array}{l}\text { BIT ERROR RATE } \\
\text { /2Mbps }\end{array}$} \\
\hline & & & $\begin{array}{l}\text { Bits } \\
\text { Error }\end{array}$ & BER (10x-6) \\
\hline 0 & 9 & 12 & 12 & 6.0 \\
\hline 1 & 0 & 0 & 0 & 0.0 \\
\hline 2 & 0 & 0 & 0 & 0.0 \\
\hline 3 & 1 & 3 & 19 & 9.5 \\
\hline 4 & 1 & 3 & 19 & 9.5 \\
\hline 5 & 9 & 12 & 12 & 6.0 \\
\hline 6 & 0 & 0 & 0 & 0.0 \\
\hline 7 & 0 & 2 & 2 & 1.0 \\
\hline 8 & 1 & 4 & 20 & 10.0 \\
\hline 9 & 0 & 0 & 0 & 0.0 \\
\hline 10 & 0 & 2 & 2 & 1.0 \\
\hline 11 & 0 & 0 & 0 & 0.0 \\
\hline 12 & 0 & 1 & 1 & 0.5 \\
\hline 13 & 1 & 5 & 21 & 10.5 \\
\hline 14 & 0 & 2 & 2 & 1.0 \\
\hline
\end{tabular}

Fuente. Elaboración propia

Los nodos de origen y destino (0 y 5), reciben 12 paquetes, con una tasa de error de bit en ambos de $6 \times 10^{-}$ 6 y los nodos que se encuentran dentro del radio de cobertura $(3,4,8$ y 13$)$ reciben en promedio 3.5 paquetes, tanto de control como de información.

Los nodos que intervienen en el establecimiento del enlace, intercambian un mayor número de paquetes que los demás, y los nodos con cero paquetes recibidos (1, 2, 6 y 9), se interpretan como nodos inactivos.

\subsection{Caso 2. Nodo fuera del alcance del origen.}

En la tabla $\mathrm{V}$ se recopilan los resultados de las simulaciones, utilizando la misma configuracion en la red de comunicaciones que en el caso 1 . Se aprecian el números de bits erróneos (BER), junto al número de paquetes recibidos y aceptados por cada host AODV.

Según la figura 6, los nodos de origen y destino (0 y 2), reciben ahora 27 y 18 paquetes, respectivamente, con una tasa de error de bit de $18 \times 10^{-6}$ y $12 \times 10^{-6}$. Los nodos intermedios ubicados en la trayectoria entre el orígen $\mathrm{y}$ 
destino $(3,7,8,10$ y 13$)$, reciben en promedio 40 paquetes, tanto de control como de información.

TABLA V

RESULTADOS CASO 2. NODO FUERA DEL ALCANCE DEL NODO ORÍGEN

\begin{tabular}{|c|c|c|c|c|}
\hline \multicolumn{5}{|c|}{$\begin{array}{l}\text { NODO FUERA DE ALCANCE } \\
\text { (host } 0 \text { a host } 2 \text { ) }\end{array}$} \\
\hline \multirow{2}{*}{$\begin{array}{l}\text { HOST } \\
\text { AODV }\end{array}$} & \multirow{2}{*}{$\begin{array}{c}\text { PAQUETES } \\
\text { RECIBIDOS } \\
\text { EXITOSOS } \\
\text { RCVDPK }\end{array}$} & \multirow{2}{*}{$\begin{array}{l}\text { PAQUETES } \\
\text { RECIBIDOS }\end{array}$} & \multicolumn{2}{|c|}{$\begin{array}{l}\text { BIT ERROR RATE } \\
\text { /2Mbps }\end{array}$} \\
\hline & & & $\begin{array}{c}\text { Error } \\
\text { Bits } \\
\end{array}$ & BER (10-6) \\
\hline 0 & 17 & 27 & 36 & 18.0 \\
\hline 1 & 3 & 12 & 12 & 6.0 \\
\hline 2 & 16 & 18 & 25 & 12.5 \\
\hline 3 & 14 & 42 & 67 & 33.5 \\
\hline 4 & 5 & 17 & 27 & 13.5 \\
\hline 5 & 5 & 24 & 49 & 24.5 \\
\hline 6 & 3 & 13 & 13 & 6.5 \\
\hline 7 & 4 & 35 & 71 & 35.5 \\
\hline 8 & 14 & 41 & 63 & 31.5 \\
\hline 9 & 3 & 13 & 13 & 6.5 \\
\hline 10 & 21 & 45 & 53 & 26.5 \\
\hline 11 & 4 & 16 & 16 & 8.0 \\
\hline 12 & 4 & 17 & 18 & 9.0 \\
\hline 13 & 6 & 39 & 76 & 38 \\
\hline 14 & 4 & 25 & 55 & 27.5 \\
\hline
\end{tabular}

Fuente. Elaboración propia

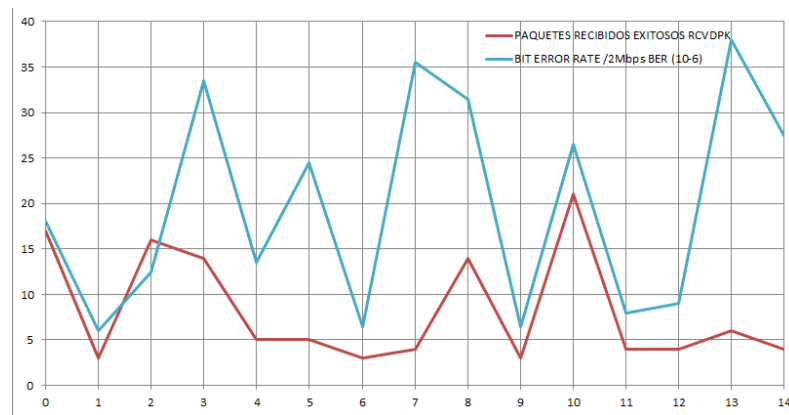

Figura 6. Caso 2, paquetes recibidos exitosos y "BER" por nodo. Fuente. Elaboración propia.

\section{CONCLUSIONES}

En ésta sección, se presentan los resultados obtenidos del análisis de algoritmo AODV, aplicado a una red de sensores inalámbricos, cuyas capas física y de enlace, están basadas en el estandar IEEE 802.11, usando la herramienta de simulación OMNET++, en parámetros como el overhead, según el intercambio de paquetes entre nodos, y la tasa de error de bits.

El overhead (paquetes de configuración de la red), se evidencia de acuerdo al número de paquetes recibidos por los nodos que no participan en el enlace establecido, debido a que el algoritmo inunda la red con los mensajes RREP, que son reenviados en respuesta a un mensaje de solicitud RREQ:

- En el caso 1, el nodo destino se encuentra al alcance del nodo origen. No existen nodos intermedios, pero los nodos 3, 4, 8 y 13 reciben un paquete RREQ.

- Para el caso 2, los nodos $(1,4,5,7,9,11,12,13$, $14)$, reciben en promedio 40 paquetes, por lo que se aprecia su incremento, para un enlace de 5 nodos intermedios $(3,7,8,10$ y 13$)$.

- La tasa de error de bit es 5.4 veces mayor en el caso 2 , teniendo en cuenta que se está trabajando sobre Wi-Fi, a una tasa de transferencia de $2 \mathrm{Mbps}$,

AODV es un algoritmo eficiente después de establecida la comunicación entre dos nodos. Sin embargo, mientras se realizan los procesos de descubrimiento y búsqueda de rutas, el overhead (mensajes de control), aumenta considerablemente, junto a la tasa de error de bit. Puede aplicarse sobre $\mathrm{Wi}-\mathrm{Fi}$, en microrredes con un número de nodos reducido, pero si aumentan, es necesario trabajar con un algoritmo de encaminamiento distinto, sobre otra tecnología a nivel de las capas física y de enlace, especialmente si se busca una modificación de los mensajes de control y busqueda de rutas, para reconfigurar la topología de la red, y poder modelar el sistema de comunicaciones adecuado.

\subsection{TRABAJOS FUTUROS}

Los resultados y conclusiones del presente artículo, buscan apoyar el diseño de un sistema de comunicaciones para una microrred que funciona en modo "Isla", donde cada generador y carga poseen un nodo sensor que intercambia datos de control en una red de sensores inalámbricos.

El análisis de los protocolos de encaminamiento para redes de sensores inalámbricos, ofrece la posibilidad de modificar sus parámetros y adaptarlos a distintos entornos de red, según la aplicación requerida. 
Lo anterior indica que, el algoritmo de encaminamiento puede modificarse para mejorar el desempeño de una red de sensores y mejorar su tiempo de vida, por lo que se espera trabajar con otros algoritmos, como DYMO, y tecnologías de transmisión, como el estándar IEEE 802.15.4, para obtener un esquema de comunicaciones apropiado en una microrred eléctrica.

\section{REFERENCIAS}

[1] M. A. Hussain, P. Khan, and Kwak kyung Sup, "WSN Research Activities for Military Application," Adv. Commun. Technol. 2009. ICACT 2009. 11th Int. Conf., vol. 01, pp. 271274, 2009.

[2] M. T. Lazarescu, "Design of a WSN platform for long-term environmental monitoring for IoT applications," IEEE J. Emerg. Sel. Top. Circuits Syst., vol. 3, no. 1, pp. 45-54, 2013.

[3] S. W. Nourildean, "A Study of ZigBee Network Topologies for Wireless Sensor Network with One Coordinator and Multiple Coordinators," Tikrit J. Eng. Sci., vol. 19, no. 4, pp. 65-81, 2012.

[4] M. Simek and P. Moravek, "Modeling of Energy Consumption of Zigbee Devices in Matlab Tool," Elektrorevue, vol. 2, no. 3, pp. 41-46, 2011.

[5] Y. Luo, J. He, H. Liu, and L. Wu, "Application of the Distributed Generation, Micro and Smart Power Grid in the Urban Planning," no. 2012, pp. 634-637, 2014.

[6] E. E. Gaona, C. L. Trujillo, and J. A. Guacaneme, "Rural Microgrids and its potential application in Colombia," p. 29.

[7] N. D. Hatziargyriou, S. Member, I. A. Dimeas, S. M. Ieee, A. G. Tsikalakis, and S. Member, "Management of Microgrids Environment in Market."

[8] I. Akyildiz, W. Su, Y. Sankarasubramaniam, and E. Cayirci, "Wireless sensor networks: a survey," Comput. Networks, vol. 38, pp. 393422, 2002.

[9] J. R. V. Jeny and a D. Ananth, "Analysis of Routing Protocols for Wireless Sensor Networks: A Survey," Int. J. Sci. Res., vol. 2, no. 2, 2013.

[10] N. Singh, R. Dua, and V. Mathur, "Wireless Sensor Networks: Architecture, Protocols, Simulator Tool," Int. J., vol. 2, no. 5, pp. 229233, 2012.

[11] F. Yao, S. H. Yang, and W. Zheng, "Mitigating interference caused by IEEE $802.11 \mathrm{~b}$ in the IEEE 802.15.4 WSN within the environment of smart house," Conf. Proc. - IEEE Int. Conf. Syst. Man Cybern., pp. 2800-2807, 2010.
[12] Y. Yu and Y. Yao, "Improved AODV routing protocol for wireless sensor networks and implementation using OPNET," 2012 Third Int. Conf. Intell. Control Inf. Process., pp. 709-713, 2012.

[13] O. Ahmed, A. Sajid, M. A. Mehmood, and M. Sciences, "Comparison of Routing Protocols to Assess Network Lifetime of WSN," vol. 8, no. 6, pp. 220-224, 2011.

[14] A. Kumar, A. Sharma, and K. Grewal, "Resolving the paradox between IEEE 802.15.4 and Zigbee," 2014 Int. Conf. Reliab. Optim. Inf. Technol., no. Llc, pp. 484-486, 2014.

[15] D. Arora, E. Millman, and S. W. Neville, "Assessing the performance of AODV, DYMO, and OLSR routing protocols in the context of larger-scale denser MANETs," IEEE Pacific RIM Conf. Commun. Comput. Signal Process. Proc., pp. 675-679, 2011.

[16] N. Kumar, K. Vashishtha, and K. Babu, "A Comparative Study of AODV , DSR , and DYMO routing protocols using OMNeT ++," no. September, pp. 735-739, 2013.

[17] D. W. Kum, J. S. Park, Y. Z. Cho, and B. Y. Cheon, "Performance evaluation of AODV and DYMO routing protocols in MANET," 2010 7th IEEE Consum. Commun. Netw. Conf. CCNC 2010, pp. 1-2, 2010.

[18] Z. Hong-tu and M. Yue-qi, "Improved Routing Algorithm Research for ZigBee Network," no. August, pp. 17-20, 2010.

[19] D. F. Ramírez and S. Céspedes, "Routing in Neighborhood Area Networks: A survey in the context of AMI communications," J. Netw. Comput. Appl., vol. 55, pp. 68-80, 2015.

[20] S. Baudoin, I. Vechiu, and H. Camblong, "A review of voltage and frequency control strategies for islanded microgrid," vol. 33, no. 0.

[21] M. R. D. Zadeh, a. Hajimiragha, M. Adamiak, a. Palizban, and S. Allan, "Design and implementation of a microgrid controller," 2011 64th Annu. Conf. Prot. Relay Eng., pp. 137145, 2011.

[22] S. Lu, M. a. Elizondo, N. Samaan, K. Kalsi, E. Mayhorn, R. Diao, C. Jin, and Y. Zhang, "Control strategies for distributed energy resources to maximize the use of wind power in rural microgrids," IEEE Power Energy Soc. Gen. Meet., pp. 1-8, 2011.

[23] A. Narayanan, P. Peltoniemi, T. Kaipia, and J. Partanen, "Energy Management System for LVDC Island Networks Acknowledgments Keywords."

[24] Y. Li and F. Nejabatkhah, "Overview of control, integration and energy management of microgrids," J. Mod. Power Syst. Clean Energy, 
vol. 2, no. 3, pp. 212-222, 2014.

[25] Z. Jiang and X. Yu, "Active power - Voltage control scheme for islanding operation of inverter-interfaced microgrids," 2009 IEEE Power Energy Soc. Gen. Meet. PES '09, pp. 17, 2009.

[26] T. L. Vandoorn, J. D. M. De Kooning, B. Meersman, and L. Vandevelde, "Review of primary control strategies for islanded microgrids with power-electronic interfaces," Renew. Sustain. Energy Rev., vol. 19, pp. 613628, 2013.

[27] E. Egea-Lopez, "Simulation tools for wireless sensor networks," Proc. ..., pp. 2-9, 2005.

[28] P. Neves, J. Fonsec, and J. Rodrigue, "Simulation tools for wireless sensor networks in medicine: a comparative study," Int. Jt. Conf. ..., vol. 2, no. January, 2007.

[29] J. Pan, "A Survey of Network Simulation Tools: Current Status and Future Developments," pp. $1-13,2008$.

[30] "OMNeT++ Discrete Event Simulation System." [Online]. Available: https://omnetpp.org/doc/omnetpp/manual.

[31] "OPNET IT Guru Academic Edition." [Online]. Available:

www.opnet.com/university_program/itguru_aca demic_edition.

[32] V. K. Taksande and K. D. Kulat, "A simulation comparison among AODV, DSDV, DSR protocol with IEEE 802.11 MAC for grid topology in MANET," Proc. - 2011 Int. Conf. Comput. Intell. Commun. Syst. CICN 2011, vol. 1, pp. 63-67, 2011. 